\title{
Ocular manifestations of mitochondrial disease
}

\section{SD Mathebula}

\author{
Department of Optometry, University of Limpopo, Private Bag X1106, Sovenga, 0727 South Africa
}

Received 1 August 2011; revised version accepted 10 February 2012

<solani.mathebula@ul.ac.za>

\begin{abstract}
Mitochondrial disease caused by mutations in mitochondrial DNA is recognized as one of the most common causes of inherited neurological disease. Neuro-ophthalmic manifestations are a common feature of mitochondrial disease. Optic atrophy causing central visual loss is the dominant feature of mitochondrial DNA diseases. Nystagmus is also encountered in mitochondrial disease.

Although optometrists are not involved with the management of mitochondrial disease, they are likely to see more patients with this disease. Ophthalmic examination forms part of the clinical as-
\end{abstract}

sessment of mitochondrial disease. Mitochondrial disease should be suspected in any patient with unexplained optic neuropathy, ophthalmoplegia, pigmentary retinopathy or retrochiasmal visual loss. Despite considerable advances in the understanding of mitochondrial genetics and the pathogenesis of mtDNA diseases, no effective treatment options are currently available for patients with mitochondrial dysfunction. (S Afr Optom 2012 71(1) 46-50)

Key Words: dominant optic atrophy, genes, Leber hereditary optic neuropathy, mitochondrial disease, DNA mutation, neuro-ophthalmology.

\section{Introduction}

Mitochondrial diseases are a group of genetic disorders that affect organs which depend a lot on aerobic metabolism ${ }^{1-4}$. They are recognized as a common cause of metabolic disease. The most common neuroophthalmic manifestations of mitochondrial disease are bilateral optic neuropathy, ophthalmoplegia, dominant optic atrophy, Kearn-Sayre syndrome and retrochiasmal visual loss. Ocular features are not isolated and may coexist with neurological and/ or systemic symptoms ${ }^{2}$. Understanding of the neuro-ophthalmic manifestations of mitochondrial disease can help in diagnosis and treatment of patient and/ or counselling of close family members.

Optometrists are likely to see patients with mitochondrial disease in their practice, as they are the first clinician the patient consults. The purpose of this paper is to review the recent data on mitochondrial diseases with emphasis on their neuro-ophthalmic manifestations. There is still a lot to learn about the pathophysiology of mitochondrial diseases.

\section{Basic mitochondrial genetics}

Mitochondria are double-membrane cytoplasmic or subcellular organelles, present in all nucleated mammalian cells ${ }^{2-4}$. Their primary function is to support aerobic respiration and they produce more than $90 \%$ of a cell's ATP through oxidation phosphorylation (OXPHOS) and electron transfer ${ }^{2-6}$. It is stated that cells in highly metabolically tissues, such as the eye and optic nerve, cardiac system, oxidative muscles, pancreas, kidneys and liver, rely heavily on ATP and have increased numbers of mitochondria ${ }^{7}$. Although the production of ATP by OXPHOS is an essential task of mitochondria in the cell, other mitochondrial processes include the detoxification of reactive oxygen species (ROS), regulation of cellular apoptosis, the fission and fusion of organelle membranes among mitochondrial networks and aspects of iron metabolism, fatty acid oxidation and amino acid biosynthesis ${ }^{8}$. A disturbance of OXPHOS seems to be the main factor in the pathogenesis of mitochondrial disease since it generates energy.

Beside the 44 autosomes and two sex chromo- 
somes that make up human nuclear DNA (nDNA), there is a small extrachromosomal double-stranded circle (cytosine-rich light and guanine-rich heavy strands) of DNA that is essential for life called the mitochondrial genome or DNA (mtDNA) ${ }^{9}$. Mitochondria are under dual genetic control of both nDNA and mtDNA. The human mitochondrial (mtDNA) genome comprises of 16 base pairs of nucleotides which contains 37 genes $^{3,9-11}$. The heavy strand encodes 28 genes while the light strand encodes 9 genes. Thirteen of these genes encode protein subunits of respiratory complexes I, III, IV and V. Only complex II is solely composed of protein encoded by nuclear genes (nDNA). The mtDNA genome also encodes 22 mitochondrial tRNAs and 2 rRNAs that are essential for translation of mtDNA transcripts ${ }^{10,11}$. Nuclear genes encode the remaining OXPHOS components and all other proteins required for mitochondrial metabolism and maintenance, which are imported to mitochondria via specialized import system ${ }^{12}$.

\section{Concepts unique to mitochondrial genetics}

mtDNA have unique characteristics that differ considerably from the Mendelian genetics ${ }^{2}, 13$. The genome is maternally-inherited and is polyploidy, with multiple copies of the mtDNA within each mitochondrion. Several hundreds, if not thousands, of mitochondria are present per cell. Normally, all of the mitochondrial genomes within the cells of an individual are identical, a situation called homoplasmy. However, a mutation occurring in one copy of mtDNA can eventually result in heteroplasmy, which is a dual population of wild-type and mutated mtDNA coexisting within the same cell ${ }^{2,3}$. During mitosis, both mutate and wild-type mtDNA are randomly segregated to each daughter cell. This random segregation affects both expression and inheritance of the mitochondrial disease, thereby contributing to a wide range of clinical presentations seen in mtDNA disorder ${ }^{1,9}$.

The majority of deleterious mtDNA mutations are heteroplasmic, with clinical manifestation only becoming evident when the number of mutated mtDNA molecules exceeds a critical threshold. This threshold represents the incapacity of the remaining wildtype mtDNA to compensate for the mutated mtDNA, resulting in impaired OXPHOS and consequently in cellular and organ dysfunction. Commonly, the threshold is reached when $60-90 \%$ of the cell's mtDNA is mutated, but the proportion varies between organs depending on their metabolic requirements ${ }^{14}$. For most diseases, the clinical phenotype is not simply a direct consequence of the relative abundance of mutated mtDNA. Other factors, such as nuclear genetic background, age, sex and environment contribute to the disease process ${ }^{1}$. Furthermore, the mitochondrial disease may also be caused by mutations in the nuclear genes, which are involved in the synthesis of various subunits of the mitochondrial respiratorychain complexes ${ }^{3}$.

Mutations in mtDNA or nDNA may cause mitochondrial disease due to disturbed mitochondrial respiratory function. mtDNA mutations that cause mitochondrial diseases were first documented in 1988 by Holt et $a l^{15}$ and Wallace et al16 and now there are many mutations associated with oxidative phosphorylation diseases ${ }^{10}$. Over the past 20 years, mitochondrial dysfunction has been increasingly recognized as an important contributor to a range of neuromuscular and neurodegenerative diseases.

\section{Ocular features of mitochondrial disease}

\section{Bilateral optic neuropathy}

The most common mitochondrial disease with bilateral optic atrophy is Leber's hereditary optic neuropathy (LHON). LHON was the first human disease to be etiologically associated with a point mutation in mtDNA and is maternally inherited ${ }^{15-19}$. LHON target the retinal ganglion cell layer with sparing of the retinal pigment epithelium and photoreceptors ${ }^{20}$. In most LHON patients, visual loss is the only manifestation of the disease ${ }^{4}$. Why the LHON affects the retinal ganglion cells is not clear but the abnormal OXPHOS and deficient generation of ATP may play a role. It presents with painless central visual loss in one eye with the other eye becoming affected in the next two months $5,21,22$. The visual loss usually occurs between the ages of 20 and 40 and is more common in men. Since mitochondrial diseases are maternally inherited, there will be no male-to-male transmission of LHON. Affected males do not transmit the genetic mutation to any of their children. Affected or carrier women transmit their LHON mutation to all of their children. It is not known why males are more suscep- 
tible to visual loss in LHON. This gender bias could result from a combination of anatomical, hormonal and physiological variations between males and females. Pupillary functions are spared and patients report no pain on eye movements ${ }^{20}$. Funduscopic abnormalities may include hyperemia of the optic nerve head, dilation and tortuosity of vessels, haemorrhages or pseudo edema 4 . LHON does not occur alone but is associated with some neurological illness. At the moment there is no treatment proven benefit for LHON. Tobacco, alcohol consumption and head trauma has been suggested as some of the triggers to the development of LHON ${ }^{21}$. Ancillary tests are of limited value but may be used to monitor progress. Fluorescein angiography may help distinguish the LHON optic disc from disc edema. Optical coherence tomography can be used for more detailed study of retinal nerve fibre layer thickness. Three point mutations in mtDNA known as the primary LHON mutations are located at mtDNA nucleotide positions 11778, 14484 and 3460, which all involve genes encoding complex I subunits of the mitochondrial respiratory chain ${ }^{1-5}$.

\section{Dominant optic atrophy (DOA)}

Dominant optic atrophy is the most common autosomal hereditary optic neuropathy. It is characterized by a slow progressive painless, bilateral, symmetrical visual loss 2,23 . The onset of symptoms is relative insidious. DOA exhibits no gender bias unlike LHON which is more common in men. The onset is usually within the first two decades of life ${ }^{23}$. Visual loss is usually detected between the ages of four and six years. Defects in colour vision are thought to result from a generalized dyschromatopsia, involving both the blue-yellow and red-green axes, with a minority of patients having pure tritanopia ${ }^{24}$. There are central field defects. The peripheral fields are usually full. There may be optic disc cupping which might be misdiagnosed as glaucoma ${ }^{3,20}$. Retinal ganglion cells appear to be the primary target. Magnetic resonance imaging (MRI) data from patients with DOA have also show significant tissue loss and thinning of the optic nerve along its entire length ${ }^{4}$. Optical coherence tomography and visual electrophysiological tests can be carried out to exclude retinal pathology and confirm optic nerve dysfunction. The majority of cases of DOA are caused by mutations in the OPA 1 gene $^{4}$.
There is no specific treatment.

\section{Ophthalmoplegia}

This is one of the most common presentations of mtDNA in adults. It is characterized by painless bilateral progressive loss of extraocular muscle mobility leading to impaired eye movement and ptosis $^{2,5,9,24-26}$. Ptosis is frequently the presenting symptom. Patients may be unaware of their extraocular muscle limitations until the disease is severe, where the ptotic eyelids occlude the pupils interfering with vision and patients may then adopt a backward head tilt. When the patient adopts a backward head tilt, the frontalis muscles may be elevated to compensate for the ptotic eyelids occluding the pupils. Even the orbicularis oculi muscles may get involved by becoming weak and result in lagophthalmos and ectropion. This may cause exposure keratopathy ${ }^{26}$. Visual acuity is usually not affected. Pupillary function is always spared ${ }^{27}$. Pain and proptosis are not features of this disease. The typical cause of ophthalmoplgia is multiple mtDNA deletions.

\section{Kearns-Sayre syndrome (KSS)}

Kearns-Sayre syndrome is associated with the development of retinitis pigmentosa and ophthalmoparesis occurring before the age of twenty years $5,9,25$. It is also called pigmentary retinopathy. The cause is thought to be the degeneration of the retinal pigment epithelium with secondary disturbance of cones, rods and the choriocapillaris ${ }^{28}$. mtDNA is prone to damage retinal pigment epithelium cells, possibly due to a high degree of oxidant stress during phagocytosis of photoreceptor outer segments. KSS is a result of point mutations of mtDNA ${ }^{29}$. Clinical examination will show a "salt and pepper" retinopathy ${ }^{30}$. This presents as fine pigment dusting in the periphery. To evaluate this condition one will need to perform an indirect ophthalmoscopy for accurate detection.

\section{Retrochiasmal visual loss}

Patients with mitochondrial disease may have visual loss not ascribable to optic nerve or retinal dysfunction, but rather a reflection of the disruption of the retrochiasmal visual pathways. Retrochiasmal visual loss is commonly associated the MELAS syndrome ${ }^{2}$. MELAS is an abbreviation for mitochondrial en- 
cephalomyopathy with lactic acidosis and stroke-like episode. MELAS is defined as stroke-like episode in the parieto-occipital regions ${ }^{9}$ and is characterized by recurrent, abrupt attacks of headaches, vomiting, focal and generalized seizures ${ }^{4}$. It results in damage to the visual pathways and causes homonymous hemianopsia or cortical blindness ${ }^{3}$. This visual loss is not due to optic neuropathy or pigmentary retinopathy. Ophthalmoscopic examination and pupillary reflexes are normal. Patients on either ethambutol or vigabatrin should have visual fields tested at baseline and at periodic intervals ${ }^{31,32}$. If visual field defects are seen, ethambutol or vigabatrin should be discontinued.

\section{Diagnosis of mitochondrial diseases}

The clinical presentation of mitochondrial disease is varied and can occur at any stage in life ${ }^{9}$. Mitochondrial disease often presents with involvement of an unusual combination of organs. More information on mitochondrial disease can be obtained from the family history. A more detailed eye examination is necessary in which the optic nerve is involved. Optical coherence tomography could be used to measure the progression of the LHON and dominant optic atrophy 33,34 .

\section{Treatment}

No specific pharmaceutical drugs have been clearly shown in large-scale clinical trials to treat mitochondrial disease effectively ${ }^{35}$. Exercise has been tested as a treatment for mitochondrial disease and has shown some success in patients ${ }^{36}$. Endurance training of patients with mitochondrial disease has shown some benefits, including improved OXPHOS and tissue perfusion $^{36-38}$. However, these benefits are lost once the patient stops exercising. This means that the physiotherapist also has a role to play in the management of mitochondrial disease. The treating physiotherapist will advise patients on appropriate exercises. Pyruvate has been proposed as a treatment option ${ }^{39}$. So far, case reports on pyruvate therapy for mitochondrial diseases are very limited and the efficacy of this treatment still remains inconclusive. Vitamins are frequently prescribed but their effectiveness is unknown or limited 40,41 .

\section{Conclusion}

Mitochondrial dysfunction can result from abnormalities in either the mtDNA or nDNA which encode mitochondrial proteins. Diagnosis can be made on clinical grounds if ophthalmoscopic features are present. Maternal history also aids diagnosis. Molecular genetic testing is the best method to confirm if a patient has one of the DNA mutations. There is no generally accepted treatment for mitochondrial disease. For general health reasons DNA mutation carriers should be advised to limit alcohol intake and stop smoking ${ }^{19}$. The administration of various pharmacological and biochemical agents, such as vitamins, cofactors, metabolites and electron acceptors, to correct or bypass the underlying respiratory chain defect has met with limited success.

\section{References}

1. McFarland R, Taylor RW, Turnbull DM. A clinical perspective on mitochondrial disease. Lancet Neurol 20109829 840 .

2. Gorman GS, Taylor RW. Mitochondrial DNA abnormalities in ophthalmological disease. Saudi J Ophthalmol 201125 395-404.

3. Fraser JA, Biousse V, Newman NJ. The neuro-ophthalmology of mitochondrial disease. Surv Ophthalmol 201055 299-334.

4. Biousse V, Newman NJ. Neuro-ophthalmology of mitochondrial diseases. Semin Neurol 200121 275-291.

5. Tuppen HAL, Blakely EL, Turnbull DM, Taylor RW. Mitochondrial DNA mutations and human disease. Biochimica Biophysica Acta 20101797 113-128.

6. Hatefi Y. The mitochondrial electron transport and oxidative phosphorylation system. Annu Rev Biochem 198554 1015-1069.

7. Zeviani M, Di Donato S. Mitochondrial disorders. Brain 2004127 2153-2172.

8. Palmieri F. Disease caused by defects of mitochondrial carriers: a review. Biochimica Biophysica Acta 20081777 564578.

9. McFarland R, Taylor RW, Turnbull DM. The neurology of mitochondrial DNA disease. Lancet Neurol 20021343 351.

10. MITOMAP: A Human Mitochondrial Genome Database. http://www.mitomap.org. 2010.

11. Chan DC. Mitochondria: dynamic organelles in disease, aging, and development. Cell 2006125 1241-1252.

12. Mokranjac D, Neupert W. Protein import into mitochondria. Biochem Soc Trans 200533 1019-1023.

13. Taylor RW, Turnbull DM. Mitochondrial DNA mutations in human disease. Nat Rev Genet 20056 389-402. 
14. Sciacco M, Bonilla E, Schon EA, DiMauro S, Moraes CT. Distribution of wild-type and common deletion forms of mtDNA in normal and respiration-deficient muscle fibers from patients with mitochondrial myopathy. Hum Mol Genet 19943 13-19.

15. Holt IJ, Harding AE, Morgan-Hughes JA. Deletions of muscle mitochondrial DNA in patients with mitochondrial myopathies. Nature 1988331 717-719.

16. Wallace DC, Singh G, Lott MT, Hodge JA, Schurr TG, Lezza AM, Elsas LJ, Nikoskelainen EK. Mitochondrial DNA mutation associated with Leber's hereditary optic neuropathy. Science 1988242 1427-1430.

17. Newman NJ. Hereditary optic neuropathies: from mitochondria to optic nerve. Am J Ophthalmol $2005140517-$ 523.

18. Newman NJ. Leber hereditary optic neuropathy; bad habits, bad vision? Brain 2009132 2306-2308.

19. Carelli V, Ross-Cisneros FN, Sadun AA. Mitochondrial dysfunction as a cause of optic neuropathies. Prog Retin Eye Res 200423 53-89.

20. Yu-Wai-Man P, Griffiths PG, Hudson G, Chinnery PF. Inherited mitochondrial optic neuropathies. J Med Genet 200946 145-158.

21. Man PY, Turnbull DM, Chinnery PF. Leber hereditary optic neuropathy. J Med Genet 200239 162-169.

22. Horvath R, Abicht A, Shoubridge EA, Karcagi V, Rozsa C, Komoly S. Leber's hereditary optic neuropathy presenting as multiple-sclerosis-like disease of the CNS. J Neurol 2000247 65-67.

23. Kerrison JB, Biousse V, Newman NJ. Retinopathy of NARP syndrome. Arch Ophthalmol 2000118 298-299.

24. Yu-Wai-Man P, Griffiths PG, Burke A, Sellar PW, Clarke MP, Gnanaraj L, Ah-Kine D, Hudson G, Czermin B, Taylor RW, Horvath R, Chinnery PF. The prevalence and natural history of dominant optic atrophy due to OPA1 mutations. Ophthalmology 2010117 1538-1546.

25. Maceluch JA, Niedziela M. The clinical diagnosis and molecular genetics of Kearns-Sayre syndrome: a complex mitochondrial encephalomyopathy. Pediatr Endocrinol Rev 20064 117-137.

26. Van Goethem G, Martin JJ, Van Broeckhoven C. Progressive external ophthalmoplegia characterized multiple deletions of mitochondrial DNA: unravelling the pathogenesis of human mitochondrial DNA instability and the initiation of genetic classification. Neuromol Med 20033 129-146.

27. Dimauro S, Schon E. Mitochondrial respiratory-chain diseases. N Engl J Med 2003348 2656-2668.

28. McKechnie NM, King M, Lee WR. Retinal pathology in Kearns-Sayre syndrome. Br J Ophthalmol 198569 63-75.

29. Maceluch JA, Niedziela M. The clinical diagnosis and molecular genetics of Kearns-Sayre syndrome: a complex mitochondrial encephalomyopathy. Pediatr Endocrinol Rev 20064 117-137.

30. Kerrison JB, Biousse V, Newman NJ. Retinopathy of NARP syndrome. Arch Ophthalmol 2000118 298-299.

31. Willmore LJ, Abelson MB, Ben-Menachem E, Pellock JM, Shields WD. Vigabatrin. Epilepsia 200950 163-173.

32. Menon V, Jain D, Saxena R, Sood R. Prospective evaluation of visual function for early detection of ethambutol toxicity.
Br J Ophthalmol 200993 1251-1254.

33. Barboni P, Carbonelli M, Savini G, Ramos Cdo V, Carta A, Berezovsky A, Salomao SR, Carelli V, Sadun AA. Natural history of Leber's hereditary optic neuropathy; longitudinal analysis of the retinal nerve fiber layer by optical coherence tomography. Ophthalmol 2010117 623-627.

34. Milea D, Sander B, Wegener M, Jansen H, Kier B, Jorgensen TM, Lund-Anderson H, Larsen M. Axonal loss occurs early in dominant optic atrophy. Acta Ophthalmol 201088 342-346.

35. Chinnery P, Majamaa K, Turnbull D, Thornburn D. Treatment for mitochondrial disorders. Cochrane Database Syst Rev 20061 CD004426.

36. Taivassalo T, Jensen TD, Kennaway N, DiMauro S, Vissing $\mathrm{J}$, Haller RG. The spectrum of exercise tolerance in mitochondrial myopathies: a study of 40 patients. Brain 2003 126 413-423.

37. Taivassalo T, Gardner JL, Taylor RW, Taylor RW, Schaefer AM, Newman J, Barron MJ, Haller RG, Turnbull DM. Endurance training and detraining in mitochondrial myopathies due to single large-scale mtDNA deletions. Brain 2006129 3391-3401.

38. Taivassalo T, Haller RG. Exercise and training in mitochondrial myopathies. Med Sci Sport Exerc 200537 2094-2101.

39. Tanaka M, Nishigaki Y, Fuku N, Ibi T, Sahashi K, Koga Y. Therapeutic potential of pyruvate therapy for mitochondrial disease. Mitochondrion 20077 399-401.

40. Marriage B, Clandinin MT, Glerum DM. Nutritional cofactor treatment in mitochondrial disorders. $J$ Am Diet Assoc 2003103 1029-1038.

41. DiMauro S, Rustin P. A critical approach to the therapy of mitochondrial respiratory chain and oxidative phosphorylation diseases. Biochimica et Biophysica Acta 20091792 1159-1167. 